OPEN ACCESS

Edited by: Jose Antonio Lopez-Escamez, Granada University Hospital, Spain

Reviewed by: Vanesa Perez-Guillen, Hospital Universitari y Politècnic La Fe, Spain Eduardo Martin-Sanz, Hospital de Getafe, Spain

*Correspondence: Toshihisa Murofushi toshi-tky@umin.ac.jp

Specialty section: This article was submitted to Neuro-otology, a section of the journal Frontiers in Neurology

Received: 19 October 2016 Accepted: 20 April 2017 Published: 08 May 2017

Citation: Murofushi T, Tsubota M, Suizu R and Yoshimura E (2017) Is Alteration of Tuning Property in Cervical Vestibular-Evoked Myogenic Potential Specific for Ménière's Disease? Front. Neurol. 8:193. doi: 10.3389/fneur.2017.00193

\section{Is Alteration of Tuning Property in Cervical Vestibular-Evoked Myogenic Potential Specific for Ménière's Disease?}

\author{
Toshihisa Murofushi ${ }^{1 *}$, Masahito Tsubota ${ }^{1}$, Ryota Suizu ${ }^{1}$ and Eriko Yoshimura ${ }^{2}$ \\ ${ }^{1}$ Department of Otolaryngology, Teikyo University School of Medicine Mizonokuchi Hospital, Kawasaki, Japan, ${ }^{2}$ Yoshimura ENT \\ Clinic, Fujisawa, Japan
}

Objective: The aim of this study is to show sensitivity and specificity of cervical vestibularevoked myogenic potential (cVEMP) tuning property test to Ménière's disease (MD) in comparison with healthy controls $(\mathrm{HC})$ and patients with other vestibular diseases.

Subjects: Totally 92 subjects (50 women and 42 men, 20-77 years of age) were enrolled in this study. Subjects were composed of 38 definite unilateral MD patients, 11 unilateral benign paroxysmal positional vertigo patients, 14 vestibular migraine patients, 19 unilateral vestibular neuritis patients, and $10 \mathrm{HC}$.

Methods: The subjects underwent cVEMP testing to 500 and $1,000 \mathrm{~Hz}$ short tone bursts (125 dBSPL). The corrected amplitudes of the first biphasic responses (p13-n23) (cVEMP) were measured. Then, a tuning property index (the $500-1,000 \mathrm{~Hz}$ cVEMP slope) was calculated.

Results: The area of under the ROC curve (AUC) was 0.75 in comparison with other vestibular disease patients, while AUC was 0.77 in comparison with other vestibular disease patients plus HC. The best cutoff point of the $500-1,000 \mathrm{~Hz}$ cVEMP slope was -19.9 . Sensitivity of the tuning property test to MD was 0.74 , while specificity was 0.76 to other vestibular disease patients.

Conclusion: The tuning property test of CVEMP is useful as a screening test of MD.

Keywords: endolymphatic hydrops, Ménière's disease, tuning, saccule, otolith organ, vertigo

\section{INTRODUCTION}

Ménière's disease (MD) is one of representative peripheral vestibular diseases. MD is characterized by episodic vertigo attacks, fluctuating hearing loss, tinnitus, and aural fullness. Although its exact pathophysiology remains unclear, MD has been recognized as an idiopathic syndrome of endolymphatic hydrops (EH) (1). A method for detecting EH using magnetic resonance (MR) imaging has been developed (2). MR imaging requires high costs and is currently used in a research setting (3). Therefore, the physiological confirmation of EH is still useful for diagnosis of EH in MD (4). For detection of EH in the cochlea, electrocochleography (5) and glycerol test using improvement of pure-tone hearing as an index (6) have been used, while for detection of $\mathrm{EH}$ in the semicircular 
canal, furosemide test has been used (7). However, the detection methods of EH in the otolith organs including the saccule have been rarely reported.

Recently, Murofushi et al. reported methods for detection of $\mathrm{EH}$ in the saccule, which has been reported to exhibit EH most frequently among the structures of the vestibular labyrinth $(8,9)$. The one is the glycerol cervical vestibular-evoked myogenic potential (cVEMP) test, and the other is the tuning property test of cVEMP (4). Traditionally, glycerol has been used for the test of $\mathrm{EH}$ in the cochlea because glycerol temporally reduces $\mathrm{EH}$ in the inner ear (6). Then, improvement of pure-tone hearing after glycerol administration was regarded as a sign suggestive of $\mathrm{EH}$ in the cochlea. Recently, glycerol administration has been applied to cVEMP test, a test of functions of the saccule, to detect EH in the saccule (4). As glycerol cVEMP test, improvement of cVEMP responses was assessed after intravenous infusion of $10 \%$ glycerol $(500 \mathrm{ml}, 2 \mathrm{~h})$ (4). The positivity rate (PR) of glycerol cVEMP test was $60 \%(12 / 20)$ in definite MD patients when significant improvement of cVEMP amplitude (p13-n23) was adopted as an index.

The tuning property test is another test for detection of $\mathrm{EH}$ in the saccule. As Rauch et al. reported (10), MD patients tend to show $1,000-\mathrm{Hz}$ dominant cVEMP responses in comparison with cVEMP responses to $500 \mathrm{~Hz}$, while healthy subjects show $500-\mathrm{Hz}$ dominant cVEMP responses. Murofushi et al. calculated $500-1,000 \mathrm{~Hz}$ cVEMP slope to quantify this frequency preference (see Methods in this article) (11). According to Murofushi et al. (4), PR of tuning property test in definite MD ears was $69 \%$ $(11 / 16)$ when totally cVEMP-absent patients were excluded. In that study, the cutoff point was set using mean - 2SD of the healthy subjects. Furthermore, results of the tuning property test almost corresponded to those of the glycerol cVEMP test except for patients with no cVEMP response. The tuning property test, which only requires recording cVEMP to 500 and $1,000 \mathrm{~Hz}$ short tone bursts (STBs), seems to be practically useful as an easy way of $\mathrm{EH}$ detection in the saccule because physiological EH detection test has never been reported except for glycerol cVEMP test, which is much more time consuming than tuning property test. Although PR of tuning property test ( $=69 \%$ in the previous study) (4) sounds low, it should be noted that $\mathrm{EH}$ in the vestibule was detected only in $70 \%$ of definite MD patients even though gadolinium-enhanced MRI was applied (12).

Although the tuning property test seems to be able to differentiate MD patients from healthy controls (HC) (11), its specificity in comparison with other types of vestibular diseases such as benign paroxysmal positional vertigo (BPPV) and vestibular migraine (VM) is still unclear. Herein, we studied sensitivity and specificity of cVEMP tuning property test to MD in comparison with patients with other vestibular diseases.

\section{MATERIALS AND METHODS}

\section{Subjects}

Totally 92 subjects (50 women and 42 men, 20-77 years of age) were enrolled in this study. Subjects were composed of 38 definite unilateral MD patients (AAO-HNS 1995) (1), 11 unilateral BPPV patients (13), $14 \mathrm{VM}$ patients (14), 19 unilateral vestibular neuritis (VN) patients (15), and 10 healthy volunteers (Table 1). Healthy volunteers did not have medial history of otological, audiological, or vestibular disorders. Although we recruited MD patients according to AAO-HNS 1995 criteria (1), all the definite MD patients in this study fulfilled diagnostic criteria published by Barany Society in 2015 (16) as well. Although BPPV patients were recruited by traditional diagnostic criteria, all the BPPV patients in this study fulfilled diagnostic criteria published by Barany Society in 2015 (13).

\section{Methods}

The cVEMP recording was conducted as follows $(4,15)$. The recording was basically performed according to the international guidelines for cVEMP recording (17). The Neuropack system (Nihon Kohden Co. Ltd., Japan) was used to record the cVEMP. Electrodes were placed on the upper half of each sternocleidomastoid muscle (SCM), with a reference electrode placed on the lateral end of the upper sternum and a ground electrode placed on the nasion. During the recording procedure, the subjects were asked to lie in the supine position and raise their heads to contract the SCM. As acoustic stimuli, air-conducted 500 and $1,000 \mathrm{~Hz}$ STBs ( $125 \mathrm{dBSPL}$, rise $/$ fall time $=1 \mathrm{~ms}$, plateau time $=2 \mathrm{~ms}$ ) were presented through headphones (type DR-531, Elega Acoustic Co. Ltd., Japan) at a stimulation rate of $5 \mathrm{~Hz}$. The signals were amplified and bandpass filtered $(20-2,000 \mathrm{~Hz})$, and 100 responses were averaged. The time window for the recording ran from -20 to $80 \mathrm{~ms}$. To confirm the reproducibility of the results, two runs were performed for each ear. The first biphasic responses (p13-n23) produced by the SCM ipsilateral to the stimulated ear were assessed. To eliminate the effects of variations in muscle activity, the mean background amplitude was calculated from the mean rectified background activity during the 20-ms prestimulus period. The corrected (normalized) cVEMP amplitude (dimensionless) was calculated as follows and employed for comparisons.

\section{Corrected (normalized) amplitude \\ $=($ raw amplitude $) /$ (mean rectified background amplitude during $20 \mathrm{~ms}$ prestimulus period).}

In the tuning property test (4), the corrected (normalized) amplitude of the p13-n23 produced in response to the $500 \mathrm{~Hz}$ STB was compared with the corrected (normalized) amplitude of the p13-n23 produced in response to the $1,000-\mathrm{Hz}$ STB.

TABLE 1 | Age and gender in each group.

\begin{tabular}{lcrcc}
\hline & N (bodies) & Men & Women & Mean age \\
\hline Ménière's disease & 38 & 17 & 21 & 53.6 \\
Benign paroxysmal & 11 & 3 & 8 & 47.8 \\
positional vertigo & & & & \\
Vestibular migraine & 14 & 4 & 10 & 40.4 \\
Vestibular neuritis & 19 & 12 & 7 & 54.2 \\
Healthy controls & 10 & 6 & 4 & 35.2 \\
\hline Total & 92 & 42 & 50 & 49.0
\end{tabular}


The $500-1,000 \mathrm{~Hz}$ cVEMP slope was calculated as a tuning property index (a measure of frequency preference) as follows:

$$
\begin{aligned}
& 500-1,000 \mathrm{~Hz} \text { cVEMP slope } \\
& \quad=100 \times(\mathrm{CA} 500-\mathrm{CA} 1000) /(\mathrm{CA} 500+\mathrm{CA} 1000) .
\end{aligned}
$$

where CA500 $(1,000)=$ corrected (normalized) amplitude of the p13-n23 produced in response to the $500(1,000) \mathrm{Hz}$ STB. When both the CA500 and CA1000 were 0, the $500-1,000 \mathrm{~Hz}$ cVEMP slope was regarded as "uninformative." Here, "uninformative" implies that no information concerning tuning property was obtained because no response was observed to either frequency.

\section{RESULTS}

First, ROC curves (receiver operating characteristic curves) were constructed for assessment of accuracy of the tuning property test and confirmation of the best cutoff point for differentiation of MD (Figure 1) (18). In comparison with other vestibular disease patients, the area of under the ROC curve (AUC) was 0.75 (95\% CI 0.64-0.85). As a reference, we also constructed ROC curve in comparison with other vestibular disease patients plus HC. AUC was 0.77 (95\% CI 0.68-0.88). The best cutoff points suggested by ROC curves were -19.9 to both groups (using the Youden index) (18).

According to results of ROC curve study, the cutoff point was set as -19.9 . When the $500-1,000 \mathrm{~Hz}$ cVEMP slope was $\leq-19.9$, the tuning property test was regarded as positive, suggestive of

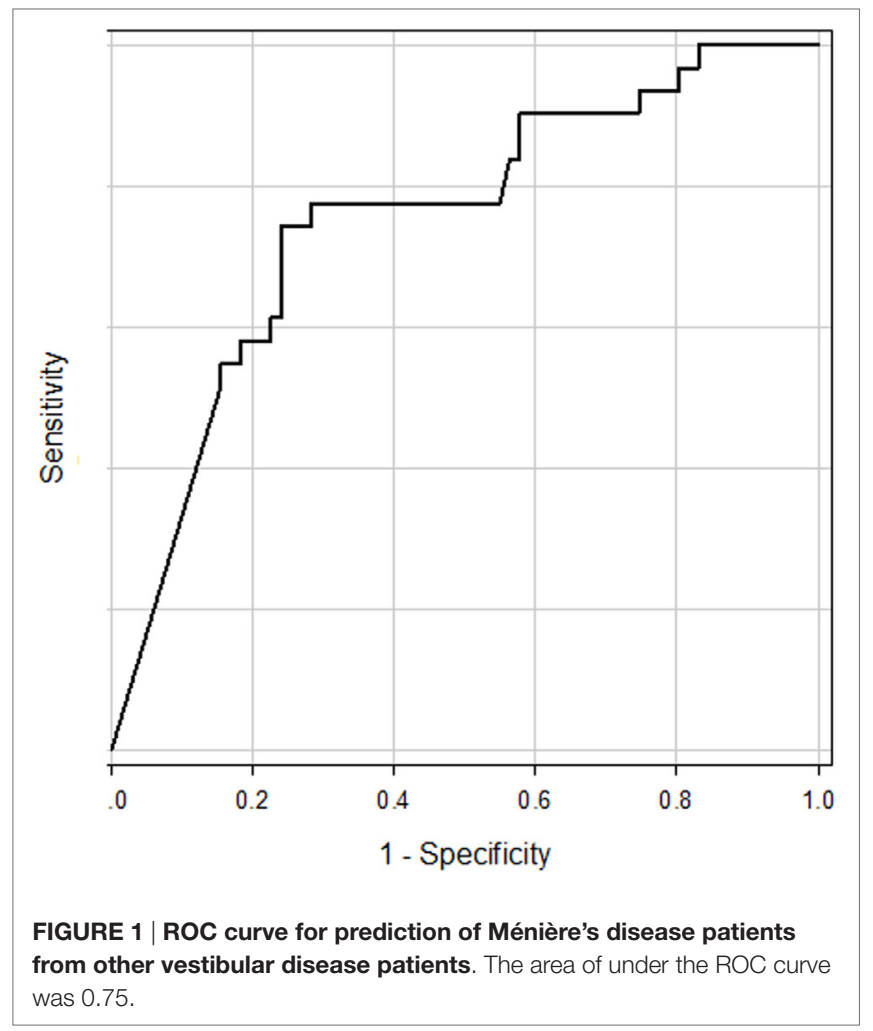

saccular EH (Figure 2). The results of the tuning property test are summarized in Table 2 and Figure 3. PR (=sensitivity of the test) was the highest in the $\mathrm{MD}$-affected ears $(\mathrm{PR}=60$ in inclusion of the uninformative as negative and 74 in exclusion of the uninformative) ( $\chi^{2}$ test, $\left.p<0.001\right)$.

In comparison between the MD-affected ears and the MDunaffected ears, PR of the MD-affected ears was significantly higher than the MD-unaffected ears $\left(\chi^{2}\right.$ test, $\left.p<0.01\right)$.

Specificities were assessed to results of ears of other vestibular disease patients and those of other vestibular disease patients plus HC, respectively (Tables 3 and 4). Specificities were 0.76 for other vestibular disease patients and 0.81 for other vestibular disease patients plus $\mathrm{HC}$.

Mean ages among the groups showed significant differences ( $p<0.001$, one-way ANOVA). Pairwise multiple comparison procedures (Holm-Sidak method) revealed that significant differences $(p<0.05)$ were observed between MD and VM, between MD and $\mathrm{HC}$, between VN and HC, and between VN and VM.

\section{DISCUSSION}

This study revealed that the tuning property test of cVEMP has moderate accuracy for differentiation of MD from patients with other vestibular diseases (and HC). To study specificity of the tuning property test not only for healthy subjects but also for patients with heterogeneous vestibular pathologies, we compared results of definite MD patients with those of such heterogeneous population, because, at clinics, we perform differential diagnoses not between MD and the healthy but between MD and other vestibular pathologies.

Furthermore, the current study suggested that the best cutoff point of 500-1,000 Hz cVEMP slope for differentiation of MD should be -19.9 . Murofushi et al. have used -19.6 as a cutoff point because it corresponded to mean - 2SD of HC (11). As a

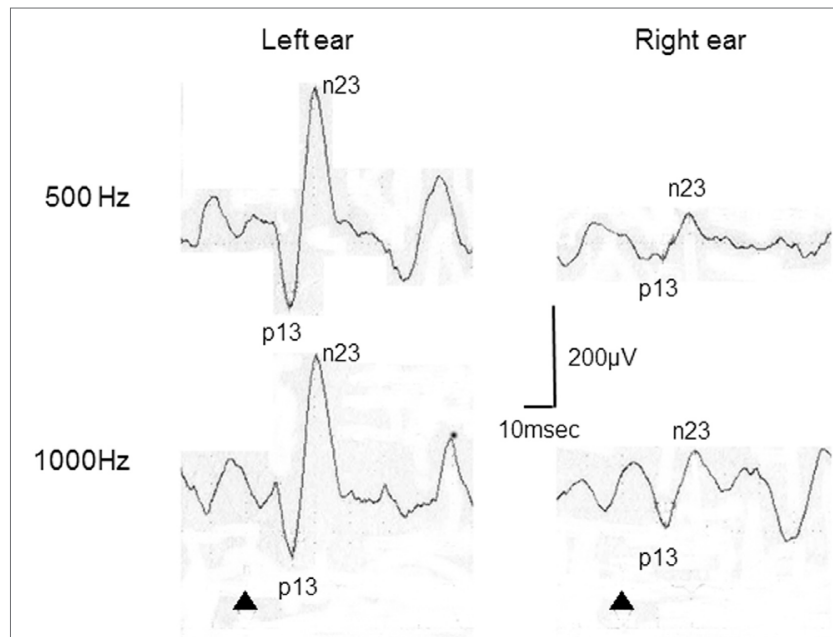

FIGURE 2 | Representative cervical vestibular-evoked myogenic potential (cVEMP) responses tuning property test positive. This patient was a 22-year-old woman with right Ménière's disease. In this case, 500-1,000 Hz cVEMP slopes were -29.8 in the right and 0 in the left. 
TABLE 2 | Positivity rate in each group.

\begin{tabular}{|c|c|c|c|c|c|c|}
\hline & $N$ (ears) & Positive $(P)$ & Negative $(N)$ & Uninformative $(U)$ & $\operatorname{PR}[100 \times P /(P+N+U)]$ & $\mathrm{PR}^{\mathrm{a}}[100 \times P /(P+N)]$ \\
\hline MD-affected side & 38 & 23 & 8 & 7 & 60.5 & 74.2 \\
\hline MD-unaffected side & 38 & 13 & 21 & 4 & 34.2 & 38.2 \\
\hline BPPV-affected side & 11 & 4 & 5 & 2 & 36.3 & 44.4 \\
\hline BPPV-unaffected side & 11 & 2 & 8 & 1 & 18.1 & 20.0 \\
\hline VM & 28 & 2 & 18 & 8 & 7.1 & 10.0 \\
\hline VN-affected side & 19 & 4 & 9 & 6 & 21.0 & 30.7 \\
\hline VN-unaffected side & 19 & 5 & 14 & 0 & 26.3 & 26.3 \\
\hline $\mathrm{HC}$ & 20 & 0 & 20 & 0 & 0 & 0 \\
\hline
\end{tabular}

$P R$, positivity rate; $M D$, Meniere's disease; BPPV, benign paroxysmal positional vertigo; VM, vestibular migraine; VN, vestibular neuritis; HC, healthy controls. ${ }^{a} P R$ in exclusion of uninformative ears.

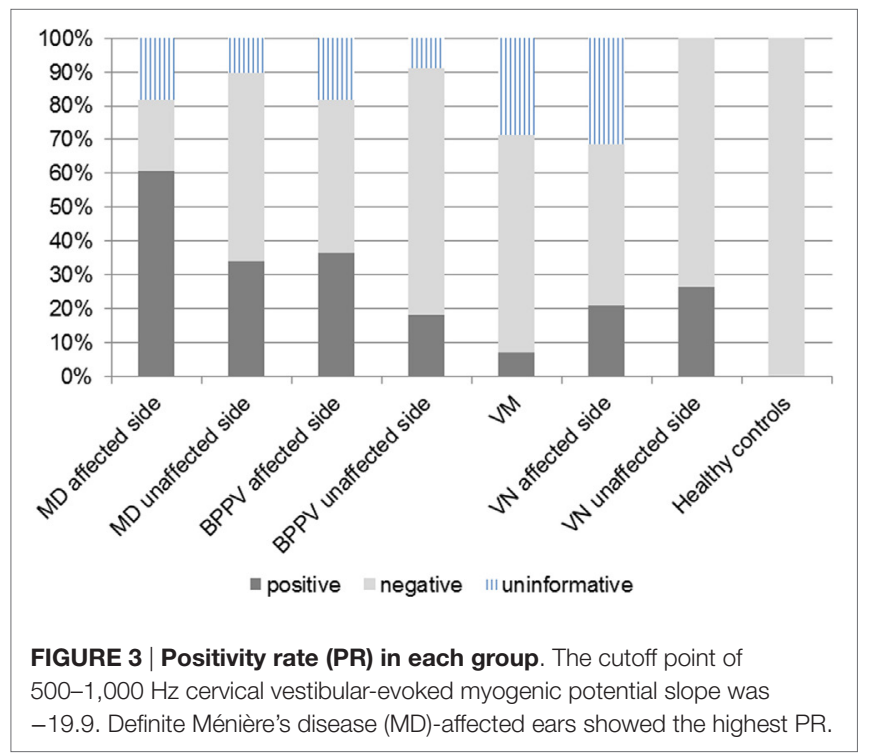

TABLE 3 | Sensitivity and specificity to other vestibular disease patients.

\begin{tabular}{lccr}
\hline & Positive & Negative & Total \\
\hline Ménière's disease-affected side & 23 & 8 & 31 \\
Other vestibular diseases & 17 & 54 & 71 \\
\hline Total & 40 & 62 & 102
\end{tabular}

TABLE 4 | Sensitivity and specificity to other vestibular disease patients plus healthy controls (HC).

\begin{tabular}{lcrr}
\hline & Positive & Negative & Total \\
\hline Ménière's disease-affected side & 23 & 8 & 31 \\
Other vestibular diseases plus HC & 17 & 74 & 91 \\
\hline Total & 40 & 82 & 122
\end{tabular}

consequence, the cutoff point suggested by ROC in this study was very close to the value used in the previous study $(-19.6)(4,11)$.

Rauch et al. reported that patients with MD exhibit a different cVEMP tuning pattern from healthy subjects (10). In their study, the healthy subjects showed best cVEMP responses around $500 \mathrm{~Hz}$, while the MD patients exhibited greater responses at $1,000 \mathrm{~Hz}$ than at $500 \mathrm{~Hz}$ on the affected side. Node et al. found that the same frequency preference observed in MD patients was normalized by furosemide loading (19). These studies suggested that an altered cVEMP tuning pattern could be a marker of saccular EH. Murofushi et al. proposed that the 500-1,000 Hz cVEMP slope could be used as an index of saccular EH $(4,11,20,21)$. Kim-Lee et al. reported the similar tendency in MD patients (22). Hereafter, this tuning pattern test is referred to as the "tuning property test" (4). Similar alteration of tuning property was also reported in ocular VEMP (oVEMP) (23).

Although previous studies have suggested difference of cVEMP tuning property between MD patients and $\mathrm{HC}$, one of clinically important points is differentiation of $\mathrm{MD}$ from other vestibular diseases. This study showed that the tuning property test has moderate accuracy for differentiation of MD patients from patients with other vestibular diseases (AUC $=0.75$, specificity $=0.76$ for -19.9 as the cutoff point).

Differentiation between MD and VM is sometimes difficult. The tuning property test could be a useful diagnostic tool for this differentiation. According to Nakada et al. (24), two of seven patients with VM showed vestibular EH in gadolinium-enhanced MRI. Their findings imply that specificity of gadolinium-enhanced MRI of MD in comparison with VM was 0.71 . VM patients with positive results in the tuning property test or gadoliniumenhanced MRI might be diagnosed as MD/VM overlapping syndrome in the future (16).

Another point of interest of this study was a moderate PR of BPPV patients in the tuning property test. It was reported that patients with BPPV could have abnormal cVEMP and oVEMP probably due to some pathological changes in the otolith organs $(25,26)$. It is well known that MD patients could present BPPVlike vertigo attacks $(27,28)$. According to a report of Hughes and Proctor, 45 (29.8\%) of 151 patients had an associated diagnosis of $\mathrm{MD}$ in a retrospective review of a large population of BPPV patients (29). Therefore, BPPV-like vertigo attacks might be the first presentation of vertigo due to $\mathrm{EH}$, followed by the development of MD. Concerning association of MD with BPPV, it was also proposed that both diseases might be caused by detached otoconia (30).

While the tuning property test is an easy way for detection of saccular $\mathrm{EH}$, it has limitations. The tuning property test does not provide any information concerning $\mathrm{EH}$ when the subjects showed absence of cVEMP to both 500 and 1,000 Hz STB. In these cases, the glycerol cVEMP test is required (4). Bilateral absence of cVEMP in the elderly could be due to aging (31). 
Secondary, the mean ages among some groups showed significant differences. These age differences might affect results. However, PR of the MD-affected ears was significantly higher than the MD-unaffected ears. It is unlikely that age differences might be a major factor of results. Third, the number of subjects in each subgroup of other vestibular diseases was limited. As a next step, a larger-sized study will be required.

\section{ETHICS STATEMENT}

Informed consent was obtained from each subject, and ethical approval was received from the ethics committee of Teikyo University (TR14-098, 15 October 2014).

\section{REFERENCES}

1. AAO-HNS guidelines for the evaluation of therapy in Meniere's disease. Otolaryngol Head Neck Surg (1995) 113:181-5.

2. Naganawa S, Yamazaki M, Kawai H, Bokura K, Sone M, Nakashima T. Visualization of endolymphatic hydrops in Ménière’s disease after single-dose intravenous gadolinium-based contrast medium: timing of optimal enhancement. Magn Reson Med Sci (2012) 11:43-51. doi:10.2463/mrms.11.43

3. Nakashima T, Pyykko I, Arroll MA, Casselbrant ML, Foster CA, Manzoor NF, et al. Meniere's disease. Nat Rev Dis Primers (2016) 2:16028. doi:10.1038/nrdp. 2016.28

4. Murofushi T, Komiyama S, Suizu R. Detection of saccular endolymphatic hydrops in Ménière's disease using a modified glycerol cVEMP test in combination with the tuning property test. Otol Neurotol (2016) 37:1131-6. doi:10.1097/MAO.0000000000001149

5. Gibson WP, Prasher DK, Kilkenny GP. Diagnostic significance of transtympanic electrocochleography in Menière's disease. Ann Otol Rhinol Laryngol (1983) 92:155-9. doi:10.1177/000348948309200212

6. Klockhoff I, Lindblom U. Endolymphatic hydrops revealed by glycerol test. Preliminary report. Acta Otolaryngol (1966) 61:459-62. doi:10.3109/ 00016486609127084

7. Futaki T, Kitahara M, Morimoto M. A comparison of the furosemide and glycerol tests for Meniere's disease. With special reference to the bilateral lesion. Acta Otolaryngol (1977) 83:272-8. doi:10.3109/00016487709128845

8. Pender DJ. Endolymphatic hydrops and Ménière’s disease: a lesion metaanalysis. J Laryngol Otol (2014) 128:859-65. doi:10.1017/S0022215114001972

9. Okuno T, Sando I. Localization, frequency, and severity of endolymphatic hydrops and the pathology of the labyrinthine membrane in Menière's disease. Ann Otol Rhinol Laryngol (1987) 96:438-45.doi:10.1177/000348948709600418

10. Rauch SD, Zhou G, Kujawa SG, Guinan JJ, Herrmann BS. Vestibular evoked myogenic potentials show altered tuning in patients with Meniere's disease. Otol Neurotol (2004) 25:333-8. doi:10.1097/00129492-200405000-00022

11. Murofushi T, Ozeki H, Inoue A, Sakata A. Does migraine-associated vertigo share a common pathophysiology with Meniere's disease? Study with vestibular evoked myogenic potential. Cephalalgia (2009) 29:1259-66. doi:10.1111/j. 1468-2982.2009.01860.x

12. Okumura $\mathrm{T}$, Imai $\mathrm{T}$, Takimoto $\mathrm{Y}$, Takeda $\mathrm{N}$, Kitahara $\mathrm{T}$, Uno $\mathrm{A}$, et al. Assessment of endolymphatic hydrops and otolith function in patients with Meniere's disease. Eur Arch Otorhinolaryngol (2017) 274:1413-21. doi:10.1007/ s00405-016-4418-2

13. von Brevern M, Bertholon P, Brandt T, Fife T, Imai T, Nuti D, et al. Benign paroxysmal positional vertigo: diagnostic criteria. J Vestib Res (2015) 25:105-17. doi:10.3233/VES-150553

14. Lempert T, Olesen J, Furman J, Waterston J, Seemungal B, Carey J, et al. Vestibular migraine: diagnostic criteria. J Vestib Res (2012) 22:167-72. doi:10.3233/ VES-2012-0453

15. Murofushi T, Nakahara H, Yoshimura E, Tsuda Y. Association of airconducted sound oVEMP findings with cVEMP and caloric test findings in patients with unilateral peripheral vestibular disorders. Acta Otolaryngol (2011) 131:945-50. doi:10.3109/00016489.2011.580003

\section{AUTHOR CONTRIBUTIONS}

All authors contributed extensively to the work presented in this paper. All authors collected data. TM wrote the manuscript. MT, RS, and EY reviewed and edited the manuscript.

\section{FUNDING}

This study was partly supported by a Grant-in-Aid from the Japan Agency for Medical Research and Development (16ek0109147h0002) and a Grant-in-Aid for Scientific Research (C) from the Japan Society for the Promotion of Science (15K10764).

16. Lopez-Escamez JA, Carey J, Chung WH, Goebel JA, Magnusson M, Mandala M, et al. Diagnostic criteria for Meniere's disease. J Vestib Res (2015) 25:1-7. doi:10.3233/VES-150549

17. Papathanasiou ES, Murofushi T, Akin FW, Colebatch JG. International guidelines for the clinical application of cervical vestibular evoked myogenic potentials: an expert consensus report. Clin Neurophysiol (2014) 125:658-66. doi:10.1016/j.clinph.2013.11.042

18. Akobeng AK. Understanding diagnostic test 3: receiver operating characteristic curves. Acta Paediatr (2007) 96:644-7. doi:10.1111/j.1651-2227.2006. 00178.x

19. Node M, Seo T, Miyamoto A, Adachi A, Hashimoto M, Sakagami M. Frequency dynamic shift of vestibular evoked myogenic potentials in patients with endolymphatic hydrops. Otol Neurotol (2005) 26:1208-13. doi:10.1097/01. mao.0000176172.87141.5d

20. Murofushi T, Komiyama S, Hayashi Y, Yoshimura E. Frequency preference in cervical vestibular evoked myogenic potential of idiopathic otolithic vertigo. Does it reflect otolithic endolymphatic hydrops? Acta Otolaryngol (2015) 135:995-9. doi:10.3109/00016489.2015.1022834

21. Murofushi T. Clinical application of vestibular evoked myogenic potential (VEMP). Auris Nasus Larynx (2016) 43:367-76. doi:10.1016/j.anl.2015. 12.006

22. Kim-Lee Y, Ahn JH, Kim YK, Yoon TH. Tone burst vestibular evoked myogenic potentials: diagnostic criteria in patients with Meniere's disease. Acta Otolaryngol (2009) 129:924-8. doi:10.1080/00016480802495412

23. Maxwell R, Jerin C, Gurkov R. Utilisation of multi-frequency VEMPs improves diagnostic accuracy for Meniere's disease. Eur Arch Otorhinolaryngol (2017) 274:85-93. doi:10.1007/s00405-016-4206-Z

24. Nakada T, Yoshida T, Suga K, Kato M, Otake H, Kato K, et al. Endolymphatic space size in patients with vestibular migraine and Meniere's disease. J Neurol (2014) 261:2079-84. doi:10.1007/s00415-014-7458-9

25. Nakahara H, Yoshimura E, Tsuda Y, Murofushi T. The damaged utricular function clarified by oVEMP in patients with benign paroxysmal positional vertigo. Acta Otolaryngol (2013) 133:144-9. doi:10.3109/00016489.2012. 720030

26. Xu F, Liang FY, Chen L, Song XC, Tong MC, Thong JF, et al. Evaluation of the utricular and saccular function using oVEMPs and cVEMPs in BPPV patients. J Otolaryngol Head Neck Surg (2016) 45:12. doi:10.1186/s40463-016-0125-7

27. Morita N, Creoglu S, Nomiya S, Nomiya R, Joglekar SS, Harada T, et al. Potential cause of positional vertigo in Meniere's disease. Otol Neurotol (2009) 30:956-60. doi:10.1097/MAO.0b013e3181b24368

28. Taura A, Funabiki K, Ohgita H, Ogino E, Torii H, Matsunaga M, et al. One-third of vertiginous episodes during the follow-up period are caused by benign paroxysmal positional vertigo in patients with Meniere's disease. Acta Otolaryngol (2014) 134:1140-5. doi:10.3109/00016489.2014.936624

29. Hughes CA, Proctor L. Benign paroxysmal positional vertigo. Laryngoscope (1997) 107:607-13. doi:10.1097/00005537-199705000-00010

30. Hornibrook J, Bird P. A new theory for Meniere's disease: detached otoconia. Otolaryngol Head NeckSurg(2016) 156:350-2.doi:10.1177/0194599816675843

31. Piker EG, Baloh RW, Witsell DL, Garrison DB, Lee WT. Assessment of the clinical utility of cervical and ocular vestibular evoked myogenic potential 
testing in elderly patients. Otol Neurotol (2015) 36:138-44. doi:10.1097/ MAO.0000000000000793

Conflict of Interest Statement: The authors declare that the research was conducted in the absence of any commercial or financial relationships that could be construed as a potential conflict of interest.
Copyright $\odot 2017$ Murofushi, Tsubota, Suizu and Yoshimura. This is an open-access article distributed under the terms of the Creative Commons Attribution License (CC BY). The use, distribution or reproduction in other forums is permitted, provided the original author(s) or licensor are credited and that the original publication in this journal is cited, in accordance with accepted academic practice. No use, distribution or reproduction is permitted which does not comply with these terms. 